\title{
Noni Seed Oil Topical Safety, Efficacy, and Potential Mechanisms of Action*
}

\author{
Afa K. Palu\#, Brett J. West, C. Jarakae Jensen
}

Research and Development Department, Morinda Inc., American Fork, USA.

Email: \#afap@tni.com

Received February $7^{\text {th }}, 2012$; revised March $13^{\text {th }}, 2012$; accepted March $30^{\text {th }}, 2012$

\begin{abstract}
Background: Noni fruit and leaf juices were used to treat acne in Polynesian folk medicine. However, the potential benefit of noni seed oil (NSO) for human skin health is not as well known. Objective: The topical safety of NSO was evaluated in a repeat insult patch test with 49 adult volunteers. Comedogenicity was evaluated in 23 adolescent volunteers for four weeks. Anti-inflammatory activity was determined in-vitro with COX-2 and 5-LOX enzyme inhibition assays. Results: No reactions to NSO were observed in any of the volunteers. NSO also reduced the number of open and closed comedones in the comedogenicity test. NSO inhibited COX-2 and 5-LOX enzymes in a concentration dependent manner, but with COX-2 inhibition being more pronounced than 5-LOX. Conclusion: Noni seed oil is safe for topical use and is non-comedogenic. It also has potential anti-inflammatory activity, being a dual inhibitor of COX-2 and 5-LOX enzymes. These results indicate the potential utility of NSO for skin care applications, as well as warrant further investigation of efficacy in 5-LOX and COX-2 related pathophysiological conditions.
\end{abstract}

Keywords: Morinda citrifolia; Noni Seed Oil; COX-2; 5-LOX; Comedones; Safety

\section{Introduction}

Morinda citrifolia L. (family Rubiaceae), commonly known as noni, has been used in Polynesia for food and medicine for thousands of years [1]. The medicinal usages of noni fruit juice, flowers, leaves, bark, and roots are wellknown among the natives of Polynesia, but less is known about the potential health benefits of noni seeds and oil extracted from them [2]. Benthall reported that noni seeds were consumed as a food by the Burmese Indians [3]. Polynesians also ate noni seeds, but perhaps, to a much lesser degree than the Burmese Indians. Therefore, this use among Pacific Islanders is not well documented in the scientific literature. But older generations of Tongan were known to pick ripe noni fruit and eat them, while removing the seeds and roasting them over an open fire before consumption. This practice was not widely observed in Tonga but was likely limited to elderly members of specific villages. One of the Tongan traditional healers from the village of Ha'alalo concluded that one has to acquire the taste of roasted noni seeds to enjoy them on a regular basis [4].

Noni seeds were considered a by-product of the noni fruit juice industry until a process for extracting the seed

\footnotetext{
"Conflict of interest: All authors are employees of Morinda Inc., largest manufacturer of noni seed oil in the world.

"Corresponding author.
}

oil was recently developed [5]. Noni fruits contain 200 250 seeds [6]. However, many thousands of noni seeds are required to produce one fluid ounce of oil. With a $4 \%$ - $6 \%$ yield and a cost of more than $\$ 400$ US dollars per gallon, noni seed oil (NSO) is a precious resource. As such, NSO production is cost prohibitive with small scale production. But when linked to a large-scale noni fruitjuice operation, it is more economically feasible.

We report two human clinical trials that demonstrate the safety of topical use of NSO on human skin and its effect on comedogenicity, with potential mechanisms of action on acne. This is the first report of NSO inhibitory effects on COX-2 and 5-LOX enzymes, especially as it relates to its potential mechanism of action against acne, as well as the formal evaluation of safety and comedogenicity.

\section{Materials and Methods}

\subsection{Ethics Reviews}

The human study protocols were reviewed by an institutional ethics review committee. Signed informed consent was obtained from all volunteers prior to initiation of the studies, which were conducted in accordance with the Ethical Principles for Medical Research Involving Human Subjects as outlined in the Declaration of Helsinki 
and in OCR HIPAA Privacy.

\subsection{Noni Seed Oil Preparation}

Noni seeds were collected from discarded material from the fruit finishing process on the island of Tahiti. Several batches of seeds were washed and dried. Samples of the dried seeds were retained for compositional analysis. Following drying, the seeds were cracked and ground to 2 - $20 \mathrm{~mm}$ particle size. The flaked seeds were then pressed in a screw press to expel the oil. For greater recovery, the remaining seed cake was mixed with a foodgrade nonpolar extraction solvent at a maximum temperature of $79^{\circ} \mathrm{C}$ for $30 \mathrm{~min}$. The extract was collected and the solvent removed by flash evaporation. The extracted oil was then combined with the pressed oil to produce crude noni seed oil [5].

\subsection{Repeat Insult Patch Test}

The repeat insult patch test is a maximization test that has been used for more than 40 years to evaluate contact allergens in human subjects [7]. The inclusion criteria for the repeat insult patch test were healthy adult volunteers with no skin conditions and limited skin pigmentation which would not interfere with reaction readings. Those excluded were persons with known sensitivities to cosmetics, pregnant or lactating, or had used medication that might prevent or limit skin reactions, such as antihistamines and corticosteroids, within 30 days prior to the test. NSO was evaluated in 46 females and 3 males (ages: $46.59 \pm 15.57)$. During the induction phase, sodium lauryl sulfate (SLS) served as an adjuvant and was mixed with NSO. Occlusive application of $25 \mu \mathrm{L}$ of the mixture to the back was accomplished with a Finn Chamber and held in place for $48 \mathrm{~h}$ with Scanpor tape. For each volunteer, application of the mixture occurred 5 times at the same site on the back, with $48 \mathrm{~h}$ rest between applications. After the induction phase, the skin of the volunteers was not exposed for at least $7 \mathrm{~d}$. Following this rest period, the skin of the volunteers was exposed to NSO, without SLS, by occlusive application for $48 \mathrm{~h}$ at a site different from the one used during induction. For $72 \mathrm{~h}$ following application, subjects were evaluated for possible delayed reaction, including redness, itching, and irritation. The skin of the volunteers was scored for reactions according to the International Contact Dermatitis Research Group and under the supervision of a consulting dermatologist [8]. Reactions were scored on a scale of 0 (no reaction) to 4 (erythema, induration, and bullae).

\subsection{Comedogenicity Test}

The effects of NSO on comedone counts was evaluated in 23 ( 8 males, 15 females) healthy adolescent Caucasian subjects (ages: $15.6 \pm 1.4 \mathrm{yr}$ ) in an open label human clinical trial. Subjects were instructed to apply equal amounts of oil to the same $2 \mathrm{~cm}$ by $2 \mathrm{~cm}$ site on the left side of the forehead daily for four weeks. Application sites were visually examined by a trained technician at the initiation of the test period, under 1.5-2X magnification, as well as four weeks later. The number of comedones was recorded at each examination. Additionally, a follicular biopsy was used to quantify the open and closed comedones before and after the trial [9]. The biopsy was performed by applying a quick-setting cyanoacrylate polymer to the same site where the oil was applied. After the polymer film had set, it was gently removed from the skin, thereby extracting the contents of sebaceous follicles. The contents of the film were then examined microscopically for the presence of keratotic plugs, with total numbers being recorded. To document and confirm the visual examinations and follicular biopsies, the application areas were photographed digitally at a distance of $25 \mathrm{~cm}$. The resultant photographic magnification was $1.5 \times$. The total number of open and closed comedones for each subject, before and after application of NSO, was determined from these measurements.

\subsection{Noni Seed Oil Effects on COX-2}

NSO was evaluated for its inhibitory effect on COX-2 enzymes, according to established protocols [10,11]. Briefly, COX-2, from human recombinant insect Sf21 cells, was combined with a mixture of 0.5 and $1 \mathrm{mg} / \mathrm{mL}$ NSO in $1 \%$ DMSO, and a $0.3 \mu \mathrm{M}$ arachidonic acid-substrate. The mixture was then incubated in buffer [100 mM Tris$\mathrm{HCl}, \mathrm{pH} 7.71,1 \mathrm{mM}$ glutathione, $1 \mu \mathrm{M}$ hematin, and 500 $\mu \mathrm{M}$ phenols] for $5 \mathrm{~min}$ at $37^{\circ} \mathrm{C}$. Rofecoxib was used as a positive control. Prostaglandin E2 (PGE2) was quantified using enzyme immunoassay (EIA) and then reported as percent inhibition. Assays were run in duplicate.

\subsection{Noni Seed Oil Effects on 5-LOX}

NSO was also evaluated for its inhibitory effects on 5lipoxygenase (5-LOX) enzymes following established protocols [12,13]. Briefly, 5-LOX enzymes, obtained from human PBML cells, were added to 0.5 and $1 \mathrm{mg} / \mathrm{mL}$ concentrations of NSO in 1\% DMSO. Arachidonic acid was added to the mixture as a substrate and incubated in HBSS buffer for $15 \mathrm{~min}$ at $37^{\circ} \mathrm{C}$. Nordihydroguaiaretic acid (NDGA) was used as a positive control. Leukotriene $\mathrm{B}_{4}\left(\mathrm{LTB}_{4}\right)$ was quantified using EIA, and results were reported as percent inhibition (Table 1). Assays were run in duplicate.

\subsection{Data Analysis}

Descriptive statistics, such as the median and mean \pm 
standard deviation (SD), were calculated. Student's $t$-test, two-tailed and unequal variance, was used to comparepre and post-trial means in both trials.

\section{Results}

\subsection{Repeat Insult Patch Test}

Previously, no evidence of acute oral toxicity was observed for NSO, and it was non-genotoxic in the Salmonella typhimurium reverse mutation assay (Ames test) or the in vitro mammalian chromosomal aberration assay [14]. During the challenge phase of the repeat-insult patch test of NSO, there were no reactions in any of the 49 volunteers. These results indicate that noni seed oil presents very little risk in causing any allergic contact dermatitis.

\subsection{Comedogenic Test}

In adolescent subjects, daily application of NSO for four weeks did not increase comedone frequency or the number of acne comedones. But it did decrease the number of open and close comedones by the end of the trial (Table 2).

\subsection{Noni Seed Oil Effects on COX-2 Enzymes}

$\mathrm{NSO}$, in concentrations of 0.5 and $1 \mathrm{mg} / \mathrm{mL}$, inhibited COX-2 enzymes by $85 \%$ and $84 \%$, respectively.

\subsection{Noni Seed Oil Effects on 5-LOX Enzymes}

$\mathrm{NSO}$, in concentrations of 0.5 and $1 \mathrm{mg} / \mathrm{mL}$, inhibited 5 -LOX enzymes by $49 \%$ and $40 \%$, respectively.

\section{Discussion}

The current study showed that noni seed oil inhibits

Table 1. Mean percent inhibition of noni seed oil (NSO) on COX-2 and 5-LOX enzymes.

\begin{tabular}{ccc}
\hline NSO Concentration $(\mathrm{mg} / \mathrm{mL})$ & \multicolumn{2}{c}{ \% Inhibition } \\
\cline { 2 - 3 } & COX-2 & 5 -LOX \\
\hline 0.5 & 85 & 49 \\
1.0 & 84 & 40 \\
\hline
\end{tabular}

Table 2. Pre and post trial comedone counts in a 4 week trial of noni seed oil daily application to the forehead of adolescent volunteers.

\begin{tabular}{ccccc}
\hline & \multicolumn{2}{c}{ Open Comedone Counts } & \multicolumn{2}{c}{ Closed Comedone Counts } \\
\cline { 2 - 5 } & Baseline & End of Study & Baseline & End of Study \\
\hline Mean & 3.60 & 2.43 & 4.36 & 3.04 \\
Median & 2 & 1 & 4 & 3 \\
Std. Dev. & 6.96 & 3.68 & 3.23 & 2.18 \\
\hline
\end{tabular}

COX-2 and 5-LOX enzymes. However, the inhibition of COX-2 was more pronounced than that of the 5-LOX enzymes. Further, NSO reduced the number of open and close comedones in healthy human subjects after 4 weeks of daily application.

Even though the inhibitory effects on 5-LOX enzymes was not pronounced compared to COX-2, 5-LOX enzymes are activated in many diseases and set in motion inflammatory responses that may lead to, among other thing, acne $[15,16]$. Therefore, it is reasonable to assume that moderate inhibition of 5-LOX enzymes, in conjunction with COX-2 inhibitory activity, will ameliorate acne and inflammatory skin conditions.

We report for the first time the potential anti-inflammatory effect on NSO through dual inhibition effects of COX-2 and 5-LOX. Belch and Hill [17] demonstrated that fatty acids, such as a linoleic acid derivative, may inhibit PGE2 synthesis by COX enzymes via substrate competition. Such an effect may explain, at least in part, the inhibition of COX-2 enzymes by NSO. The possibility of the inhibitory activity being due to linoleic acid is supported by analyses published by West and colleagues [14] which showed that linoleic acid accounted for $89.9 \pm$ $19.2 \mathrm{~g} / \mathrm{kg}$ of dried noni seeds and $594.5 \pm 60.5 \mathrm{~g} / \mathrm{kg}$ of the crude seed oil. These quantities make NSO a rich source of linoleic acid. Linoleic acid reduces the size of follicular casts and micro-comedones by as much as $25 \%$ [18]. This further demonstrates the active role of the fatty acids in NSO in the anti-inflammatory and anti-comedogenic activities observed in our study.

Dual inhibition of COX-2 and 5-LOX enzymes is effective for more than just the treatment of pain and inflammation [19-21]. The current results are consistent with previous data from our laboratory which demonstrated that noni fruit juice is a selective inhibitor of COX-2 enzymes [22]. We also demonstrated that noni fruit juice was a dual inhibitor of COX-2 and 5-LOX enzymes [23]. This dual inhibition may explain the painreducing and anti-inflammatory effect of noni juice, as reported by healers and consumers. But this is a novel discovery for noni seed oil.

Some plant-derived oils have the potential to induce comedone formation [24,25]. Therefore, new vegetable oils must be examined for their comedogenic potential prior to any topical use by humans. In our studies, NSO reduced open and closed comedone counts, indicating an absence of comedogenicity. This finding suggests that NSO has little or no potential for clogging up pores of the skin when used as a cosmetic ingredient. Analyses by West and colleagues [14] showed that NSO contains linoleic and oleic acids, which are considered healthy fatty acids. In fact, oleic fatty acid can be used in cooking of foods [26-28] and for formulations of various cosmetics [29-33]. 


\section{Conclusion}

The data support the potential for noni seed oil to reduce inflammation via its dual inhibition of COX-2 and 5LOX enzymes. The fact that it reduced open and close comedones and did not cause any reaction in the repeat insult patch test indicates that it is a safe raw material for cosmetic use.

\section{Acknowledgements}

Financial support for this project was provided by Morinda Bioactives. Gratitude is also expressed to Silivia L. T. Palu from 'Uiha, Ha'apai and Viliami Palepale from Lehi, Utah for their contribution on traditional usage of noni and noni seed consumption.

\section{REFERENCES}

[1] M. Y. Wang, B. J. West, C. J. Jensen, D. Nowicki, C. Su, A. K. Palu and G. Anderson, "Morinda Citrifolia (Noni): A Literature Review and Recent Advances in Noni Research," Acta Pharmacological Sinica, Vol. 23, No. 12, 2002, pp. 1127-1141.

[2] S. L. T. Palu, "A Traditional Healer from 'Uiha, Ha'apai, Tonga, South Pacific," Personal Communication, Orem, 2004.

[3] A. P. Benthall, "The Trees of Calcutta and Its Neighborhood," Spink, Calcutta, 1984

[4] V. A. Palepale, "A Traditional Healer from Ha'alalo, Tonga, South Pacific," Personal Communication, Lehi, 2005.

[5] J. J. Wadsworth and S. P. Story, "Morinda citrifolia Oil," US Patent No. 6417157, 2002.

[6] S. C. Nelson, "Hawaiian Noni Seed Processing and Germination," 2005.

http://www.ctahr.hawaii.edu/noni/downloads/noniseeds.p df

[7] A. M. Kligman, "The Identification of Contact Allergens by Human Assay. III. The Maximization Test: A Procedure for Screening and Rating Contact Sensitizers," TheJournal of Investigative Dermatology, Vol. 47, No. 5, 1966, pp. 393-409.

[8] R. L. Rietschel and J. F. Fowler, "Fisher's Contact Dermatitis," 4th Edition, Williams \& Wilkins, Baltimore, 1995.

[9] O. H. Mills and A. M. Kligman, "The Follicular Biopsy," Dermatologica, Vol. 167, No. 2, 1983, pp. 57-63.

[10] T. D. Warner, F. Giuliano, I. Vojnovic, A. Bukasa, J. A. Mitchell and J. R. Vane, "Nonsteroid Drug Selectivities for Cyclo-Oxygenase-1 rather than Cyclo-Oxygenase-2 Are Associated with Human Gastrointestinal Toxicity: A Full in Vitro Analysis," Proceedings of the National Academy of Sciences of the USA, Vol. 96, No. 13, 1999, pp. 7563-7568. doi:10.1073/pnas.96.13.7563

[11] D. Riendeau, S. Charleson, W. Cromlish, J. A. Mancini, E. Wong and J. Guay, "Comparison of the Cyclooxygenase-1 Inhibitory Properties of Nonsteroidal Anti-In- flammatory Drugs (NSAIDs) and Selective COX-2 Inhibitors, Using Sensitive Microsomal and Platelet Assays," Canadian Journal of Physiology and Pharmacology, Vol. 75, No. 9, 1997, pp. 1088-1095. doi:10.1139/y97-130

[12] G. W. Carter, P. R. Young, D. H. Albert, J. Bouska, R. Dyer, R. L. Bell, J. B. Summers and D. W. Brooks, "5-Lipoxygenase Inhibitory Activity of Zileuton," The Journal of Pharmacology and Experimental Therapeutics, Vol. 256, No. 3, 1991, pp. 929-937.

[13] H. Safayhi, S. E. Boden, S. Schweizer and H. P. Ammon, "Concentration-Dependent Potentiating and Inhibitory Effects of Boswellia Extracts on 5-Lipoxygenase Product Formation in Stimulated PMNL," Planta Medica, Vol. 66, No. 2, 2000, pp. 110-113. doi:10.1055/s-2000-11136

[14] B. J. West, C. J. Jensen and J. Westendorf, "A New Vegetable Oil from Noni (Morinda citrifolia) Seeds," International Journal of Food Science and Technology, Vol. 43, No. 11, 2008, pp. 1988-1992. doi:10.1111/j.1365-2621.2008.01802.x

[15] P. Rubin and K. W. Mollison, "Pharmacotherapy of Diseases Mediated by 5-Lipoxygenase Pathway Eicosanoids," Prostaglandins \& Other Lipid Mediators, Vol. 83, No. 3, 2007, pp. 188-197. doi:10.1016/j.prostaglandins.2007.01.005

[16] I. Kurokawa, F. W. Danby, Q. Ju, X. Wang, L. F. Xiang, L. Xia, W. Chen, I. Nagy, M. Picardo, D. H. Suh, R. Ganceviciene, S. Schagen, F. Tsatsou and C. C. Zouboulis, "New Developments in Our Understanding of Acne Pathogenesis and Treatment," Experimental Dermatology, Vol. 18, No. 10, 2009, pp. 821-832. doi:10.1111/j.1600-0625.2009.00890.x

[17] J. J. F. Belch and A. Hill, "Evening Primrose Oil and Borage Oil in Rheumatologic Conditions," American Journal of Clinical Nutrition, Vol. 71, No. 1, 2000, pp. 352-356.

[18] C. Letawe, M. Boone and G. E. Pierard, "Digital Image Analysis of the Effects of Topically Applied Linoleic Acid on Acne Micro-Comedones," Clinical and Experimental Dermatology, Vol. 23, No. 2, 1998, pp. 56-58. doi:10.1046/j.1365-2230.1998.00315.x

[19] A. Klegeris and P. L. McGreer, "Cyclooxygenase and 5-Lipoxygenase Inhibitors Protect Against Mononuclear Phagocyte Neurotoxicity," Neurobiology of Aging, Vol. 23, No. 5, 2002, pp. 787-794. doi:10.1016/S0197-4580(02)00021-0

[20] J. Pommery, N. Pommery and J. P. Henichart, "Modification of Eicosanoid Profile in Human Blood Treated by Dual COX/LOX Inhibitors," Prostaglandins, Leukotrienes, and Essential Fatty Acids, Vol. 73, No. 6, 2005, pp. 411-417. doi:10.1016/j.plefa.2005.08.009

[21] L. Goossens, N. Pommery and J. P. Henichart, "COX2/5-LOX Dual Acting Anti-Inflammatory Drugs in Cancer Chemotherapy," Current Topics in Medicinal Chemistry, Vol. 7, No. 3, 2007, pp. 283-296. doi:10.2174/156802607779941369

[22] C. X. Su, "A New Selective COX-2 Inhibitor: Morinda citrifolia (Noni). Eicosanoids and Other Bioactive Lipids in Cancer, Inflammation and Related Diseases," 7th An- 
nual Conference, Nashville, 14-17 October 2001.

[23] A. K. Palu, C. Su, B. N. Zhou and J. Jensen, "Morinda citrifolia L.: A Dual Inhibitor of COX-2 and 5-LOX Enzymes," 5th International Conference and Exhibition on Nutraceuticals and Functional Foods, San Francisco, 7-10 November 2004.

[24] A. Valentino, M. Fimiani, R. Baiocchi, R. Bilenchi, R. Perotti, A. Castelli, M. L. Mancianti and M. Raffaelli, "Cosmetic Acne and a Test of Comedogenicity," Bollettino Della Società Italiana di Biologia Sperimentale, Vol. 60, No. 10, 1984, pp. 1845-1848.

[25] C. R. Srinivas, A. Padhee, C. Balanchandran, S. D. Shenoy, S. Acharya and K. Ramnaraya, "Comedones Induced by Coconut Oil in a Borderline Tuberculoid Lesion," International Journal of Leprosy and Other Mycobacterial Diseases, Vol. 56, No. 3, 1988, pp. 471-472.

[26] J. A. Menendez, L. R. Vellon, R. Colomer and R. Lupu, "Oleic Acid, the Main Monounsaturated Fatty Acid of Olive Oil, Suppresses Her-2/neu (erbB-2) Expression and Synergistically Enhances the Growth Inhibitory Effects of Trastuzumab (Herceptin ${ }^{\mathrm{TM}}$ ) in Breast Cancer Cells with Her-2/neu Oncogene Amplification," Annals of Oncology, Vol. 16, No. 3, 2005, pp. 359-371. doi:10.1093/annonc/mdi090

[27] V. M. Flood, K. L. Webb, E. Rochtchina, B. Kelly and P. Mitchell, "Fatty Acid Intakes and Food Sources in a Population of Older Australians," Asia Pacific Journal of Clinical Nutrition, Vol. 16, No. 2, 2007, pp. 322-330.
[28] G. Irmisch, D. Schlafke, W. Gierow, S. Herpertza and J. Richter, "Fatty Acids and Sleep in Depressed Inpatients," Prostaglandins, Leukotrienes, and Essential Fatty Acids, Vol. 76, No. 1, 2007, pp. 1-7. doi:10.1016/j.plefa.2006.09.001

[29] C. D. Daulatabad, G. M. Mulla and A. M. Mirajkar, "Ricinoleic Acid in Morinda citrifolia Seed Oil," Journal of Oil and Technologists Association, Vol. 21, No. 2, 1989, pp. 26-27.

[30] A. Kogan and N. Garti, "Microemulsions as Transdermal Drug Delivery Vehicles," Advanced Colloid and Interface Science, Vol. 123-126, 2006, pp. 369-385. doi:10.1016/j.cis.2006.05.014

[31] L. C. Zhang, J. H. Hu and L. Li, "In Vivo and in Vitro Evaluation of Essential Oils from Ligusticum chuanxiong HORT on the Transdermal Delivery of Flurbiprofen in Rabbits," Biological \& Pharmaceutical Bulletin, Vol. 29, No. 6, 2006, pp. 1217-1222. doi:10.1248/bpb.29.1217

[32] R. Jantharaprapap and R. Stagni, "Effects of Penetration Enhancers on in Vitro Permeability of Meloxicam Gels," International Journal of Pharmaceutics, Vol. 343, No. 1-2, 2007, pp. 26-33. doi:10.1016/j.ijpharm.2007.04.011

[33] G. Martin-Ezquerra, M. Sanchez-Regana and P. UmbertMillet, "Optimization of Narrow-Band UVB with a 5\% Oleic Acid Cream in the Treatment of Psoriasis," Journal of Drugs in Dermatology, Vol. 6, No. 3, 2007, pp. 290292. 